KIAS-P06029

hep-th/0607091

\title{
A Heterotic Flux Background and Calibrated Five-Branes
}

\author{
Seok Kim* and Piljin $\mathrm{Yi}^{\dagger}$ \\ School of Physics, Korea Institute for Advanced Study, \\ 207-43, Cheongryangri-Dong, Dongdaemun-Gu, Seoul 130-722, Korea
}

\begin{abstract}
We consider, in flux compactification of heterotic string theory, spacetimefilling five-branes. Stabilizing the fivebrane involves minimizing the combined energy density of the tension and a Coulomb potential associated with an internal 2-dimensional wrapping. After reviewing the generalized calibration under such circumstances, we consider a particular internal manifold based on a $T^{2}$ bundle over a conformally rescaled $K 3$. Here, we find two distinct types of wrapping. In one class, the fivebrane wraps the fibre $T^{2}$ which belongs to a cyclic homotopy group. The winding number is not extensive, yet it maps to D3-brane number under a U-duality map to type IIB side. We justify this by comparing properties of the two sides in detail. Fivebranes may also wrap a topological 2-cycle of K3, by saturating a standard calibration requirement with respect to a closed Kähler 2 -form $J_{K 3}$ of $K 3$. We close with detailed discussion on F-theory dual of these objects and related issues.
\end{abstract}

*seok@kias.re.kr

${ }^{\dagger}$ piljin@kias.re.kr 


\section{Introduction}

Over the last few years, flux compactification of string theory has proven to be a rich playing ground for connecting to real world. From building a realistic particle physics model to understanding the inflation era, it has given many new insights. Existence of landscape [1, 2, 3, 4], namely a large number of discrete vacua, stable and semi-stable [5], offers us a completely different view on our universe.

Much of these developments came from a special subclass of type IIB flux compactification, where the internal manifold is a warped Calabi-Yau [6]. General supersymmetry requirements in type IIB and IIA are known to demand much less, known as $S U(3)$-structure [7, 8, 9, 10, 11, 12, 13, and we do not yet have a clear picture of vacua in this more general setting. Notable exceptions are the so-called F-theories, which are IIB compactifications with nonuniform dilaton-axion. In particular, the classic example of F-theory on $K 3 \times K 3$ has been explored from early on with fluxes turned on 14, 15, 16, 17, 18, 47, 20]. This example is also interesting because of a known U-dual map to the heterotic side.

Flux compactification of the heterotic string theory [21] was first considered in 1986 by Strominger, who gave a complete characterization of supersymmetry requirements [22]. While the geometry is not as simple as warped Calabi-Yau, it still has an $S U(3)$ holonomy with respect to a torsionful connection, which is still simpler than the general $S U(3)$ structure manifold. The heterotic flux compactification is known to evade the usual no-go theorem against smooth flux compactification, at the cost of introducing higher order curvature term in the equation of motion and a Bianchi identity [23, 24]. It is known that the smooth compactification is available only if one stays far away from minimal embedding configuration of the gauge bundle on the internal manifold [25, 26].

No explicit solution to the heterotic system is available. However, Fu and Yau proved an existence theorem for a smooth solution whose internal manifold is a $T^{2}$ fibre bundle over the base of conformally rescaled $K 3$ [27]. It is expected that this class of solution would map to the above mentioned $K 3 \times K 3$ F-theory (or its IIB orientifold limit), under a chain of U-duality map [28]. For the first time, we have a reasonably explicit dual pair of flux compactification model which deviates significantly from the conformally Calabi-Yau examples of IIB, on which much of recent 
applications are based. The purpose of this paper is to explore this pair, largely from the heterotic side, with emphasis on putting extra structures due to fivebranes. Under the U-dual map from F/IIB side, D-branes must be emulated by fivebranes, strings, and the gauge bundle. In this respect, understanding of fivebrane in the heterotic side remains an important issue in further exploration of this class of solution.

This note is organized as follows. After a quick summary of supersymmetry condition with flux in section 2, we import the generalized calibration of M5 branes and adapt it to the heterotic string theory in section 3. Here flux acts to contribute magnetic Coulomb energy to fivebranes which modifies the problem of finding supersymmetric brane configurations significantly. Section 4 outlines Fu-Yau solution and delineates how the generalized calibration of fivebrane specializes to this background. Two types of spacetime-filling fivebranes are found. One that winds around a homotopically trivial $T^{2}$ fibre, and those which wrap certain homology 2-cycles and orthogonal to the flux. Section 5 and 6 are devoted to complete characterization of these fivebranes, including detailed issue of tadpole conditions. In particular, fivebranes winding on $T^{2}$ are identified as U-dual of D3 branes in IIB or F-theory, despite the fact that the winding number is not an extensive quantity. We show that the cyclic nature of the $T^{2}$ winding number is in fact precisely mirrored in the number of D3 branes on F/IIB side. Section 7 tidies up some loose ends associated with this U-dual map to F/IIB side, where among other things, counting of worldvolume degrees of freedom are considered with and without fluxes.

\section{Flux Compactification of Heterotic String The- ory}

We start with the bosonic part of the supergravity/super-Yang-Mills action in ten dimensions, ${ }^{*}$

$$
\begin{aligned}
\mathscr{L}=\frac{1}{2 \kappa_{10}^{2}} \sqrt{-G} \mathrm{e}^{-2 \Phi} & {\left[R(\omega)-\frac{1}{12} H_{M N P} H^{M N P}+4\left(\nabla_{M} \Phi\right)^{2}\right.} \\
+ & \left.\frac{\alpha^{\prime}}{8}\left\{\operatorname{tr}\left(F_{M N} F^{M N}\right)-\operatorname{tr}\left(R_{M N}\left(\omega_{-}\right) R^{M N}\left(\omega_{-}\right)\right)\right\}\right],
\end{aligned}
$$

${ }^{*}$ We follow [29] for conventions, except for using anti-hermitian basis for the gauge field. 
with anti-Hermitian basis for the gauge field and anti-symmetric generators for the curvature, both of the unit normalization. According to Bergshoeff et.al. [24], the curvature that appears in the last term of the action is the one with torsion $-\frac{1}{2} H$ with the convention that the connection with torsion $+\frac{1}{2} H$ appears in the supersymmetry variation of gravitino.

With supersymmetry, the metric in string frame has no warp factor,

$$
G_{M N} \mathrm{~d} x^{M} \mathrm{~d} x^{N}=\eta_{\mu \nu} \mathrm{d} x^{\mu} \mathrm{d} x^{\nu}+g_{m n} \mathrm{~d} y^{m} \mathrm{~d} y^{n}
$$

with a metric $g_{m n}$ on the compact manifold $\mathcal{M}_{6}$. Supersymmetry also implies a complex structure $J$ which is integrable,

$$
0=N_{m n}^{p}=J_{m}^{q} \nabla_{[q} J_{n]}^{p}-J_{n}^{q} \nabla_{[q} J_{m]}^{p}
$$

with respect to which the metric $g_{m n}$ is hermitian. $J$ is also parallel under a torsionful connection

$$
\nabla_{m}^{(+)} J_{n p}=0
$$

with the connection having a torsion $H / 2 .^{\dagger}$

Supersymmetry relates the gradient of the complex structure $J$ and that of the dilaton $\Phi$, and the antisymmetric tensor $H$. First, $H$ can be identified with the so-called Bismut torsion [30, 31]

$$
H_{m n p}=-3 J_{m}^{q} J_{n}^{r} J_{p}^{s} \nabla_{[q} J_{r s]}
$$

and the dilaton is related to $J$ as

$$
\nabla_{m} \Phi=\frac{3}{4} J^{n p} \nabla_{[m} J_{n p]}
$$

The relation between dilaton and $H$ can be also read off from the above,

$$
\nabla_{m} \Phi=\frac{1}{4} J_{m n} J_{p q} H^{n p q}
$$

and tells us that the non-primitive part of $H$ is fully encoded in $\mathrm{d} \Phi$.

\footnotetext{
${ }^{\dagger}$ That is, the spin connection is shifted as $\omega_{b \mu}^{a} \rightarrow \omega_{(+) b \mu}^{a}=\omega_{b \mu}^{a}+\frac{1}{2} H_{b \mu}^{a}$ in our normalization.
} 
Note that, from these, we also have

$$
0=\mathrm{d}\left(e^{-2 \Phi} J \wedge J\right)
$$

and

$$
H=-* e^{2 \Phi} \mathrm{d}\left(e^{-2 \Phi} J\right)
$$

once we make use of the hermiticity. Throughout this note, $*$ means the Hodge-star operation with respect to the six dimensional metric $g$. We will always add subscripts to signify Hodge-star operations with respect to other metrics. In particular, the latter can be rewritten as

$$
*\left(e^{-2 \Phi} H\right)=\mathrm{d}\left(e^{-2 \Phi} J\right),
$$

which automatically solves the equation of motion for $H$.

In compactifying the heterotic theory, an important topological constraint is found in the Bianchi identity of $H$, which reads,

$$
\mathrm{d} H=\frac{\alpha^{\prime}}{4}\left[\operatorname{tr}\left(R^{\prime} \wedge R^{\prime}\right)-\operatorname{tr}(F \wedge F)\right],
$$

The curvature 2-form $R^{\prime}$ is ambiguous as far as anomaly cancelation is concerned 32]. While the natural choice with the given form of the action would be that of the connection with torsion $-H / 2$ [33, 24, any shift of torsion piece is allowed 34]. Also, as elaborated in Ref.[25], a smooth and large compactification necessarily implies that $H^{2}$ is of order $\alpha^{\prime}$ so the torsion part of $R^{\prime}$ contributes only a higher order term on the right hand side. A convenient choice for $R^{\prime}$ is to take the so-called Hermitian connection, whereby the right hand side is of Hodge type $(2,2)$. Since $\mathrm{d} H$ is of Hodge type $(2,2)$ also, this choice represents a consistent truncation of this equation.

When fivebranes are present, the Bianchi identity will acquire source terms as

$$
\mathrm{d} H=\frac{\alpha^{\prime}}{4}\left[\operatorname{tr}\left(R^{\prime} \wedge R^{\prime}\right)-\operatorname{tr}(F \wedge F)-16 \pi^{2} \delta_{\text {fivebrane }}\right],
$$

which could modify the topological constraint on the gauge bundle. Another way to view this is to consider the fivebranes as singular limits of the gauge bundle, where the Hermitian Yang-Mills degenerates such that $\operatorname{tr} F \wedge F$ becomes a delta function source. The convention here is such that supersymmetric $F$ is anti-self-dual. Since we are using the anti-hermitian basis with unit normalization also, the density $\operatorname{tr} F \wedge F / 16 \pi^{2}$ integrates to a nonnegative integer against a topological 4-cycle. 


\section{Generalized Calibration for Fivebranes}

Let us consider fivebranes which are spacetime-filling in a heterotic flux compactification with a $3+1$ Minkowski spacetime intact. Such fivebranes may be alternatively considered as small instanton limits of the gauge bundle. They would wrap two-cycles in internal manifold and thus are of co-dimension 4 objects. The two worldvolume directions along $\mathcal{M}_{6}$ should span out a two-dimensional surface, which we will denote by $\Sigma$. In this note, we will consider such fivebranes in the probe limit but will be careful to maintain the tadpole cancelation conditions for consistency.

For simplicity, we will consider configurations with no worldvolume field strength turned on. These are completely characterized by the two-dimensional embedding $\Sigma$ into $\mathcal{M}_{6}$, and we are lead to the following energy functional per unit 3-volume,

$$
\left.\operatorname{vol}_{3+1}\right\lrcorner\left[\int_{\Sigma} e^{-2 \Phi} v l_{5+1}-\int_{\Sigma} B_{6}\right],
$$

to be minimized, where $B_{6}$ is a 6 -form potential dual to $B_{2}$ and $\lrcorner$ denotes exterior contraction between differential forms. The first term is the contribution from the tension of the fivebrane, which explains the presence of $e^{-2 \Phi}$, while the second is the minimal coupling which incorporates the energy due to Coulombic potential $B_{6}$. With the above general form of $H$, the dual potential is computed as

$$
d B_{6}=e^{-2 \Phi} *_{9+1} H=e^{-2 \Phi}(* H) \wedge \operatorname{vol}_{3+1}=d\left(e^{-2 \Phi} J\right) \wedge v o l_{3+1},
$$

which gives

$$
B_{6}=e^{-2 \Phi} J \wedge \operatorname{vol}_{3+1}
$$

up to an additive ambiguity of a closed 6 -form. Since

$$
\operatorname{vol}_{5+1}=\operatorname{vol}_{\Sigma} \wedge \operatorname{vol}_{3+1}
$$

the energy functional of the wrapped fivebrane, per unit 3-volume, is

$$
\mathcal{E}(\Sigma)=\int_{\Sigma} e^{-2 \Phi} \operatorname{vol}_{\Sigma}-\int \Sigma^{*}\left(e^{-2 \Phi} J\right)
$$

A stable configuration of a wrapped fivebrane is obtained only if $\mathcal{E}$ is minimized against deformation of the embedding $\Sigma$ into $\mathcal{M}_{6}$.

$$
\delta \mathcal{E}(\Sigma)=0
$$


With the current gauge choice of $B_{6}$, in particular, this energy functional is clearly nonnegative. One class of minimized solution would be obtained for $\Sigma$ 's for which we have

$$
\mathcal{E}(\Sigma)=0
$$

Here we should emphasize that the value of $\mathcal{E}$ is not necessarily the physical energy. Only its variation is important for our purpose. In a later example, we will shift the definition of the energy by a closed form, giving it a more familiar shape.

Minimization of $\mathcal{E}$ is clearly a necessary condition for supersymmetry. Here we would like to show that $\mathcal{E}=0$ is a consequence of generalized calibration; in other words, configurations with $\mathcal{E}=0$ are actually supersymmetric. For this, we will regard our fivebranes as M5 branes in the heterotic M-theory [35, 36, 37, which are orthogonal to the 11-th direction. The metric of the spacetime is

$$
\begin{aligned}
G_{A B}^{10+1} \mathrm{~d} X^{A} \mathrm{~d} X^{B} & =e^{4 \Phi / 3} \mathrm{~d} x_{11}^{2}+e^{-2 \Phi / 3} G_{M N} \mathrm{~d} x^{M} \mathrm{~d} x^{N} \\
& =e^{4 \Phi / 3} \mathrm{~d} x_{11}^{2}+e^{-2 \Phi / 3}\left(\eta_{\mu \nu} \mathrm{d} x^{\mu} \mathrm{d} x^{\nu}+g_{m n} \mathrm{~d} y^{m} \mathrm{~d} y^{n}\right)
\end{aligned}
$$

for an interval along $x_{11}$, while there is nontrivial 3 -form potential $C_{3}$

$$
\mathrm{d} C_{3}=d x_{11} \wedge \frac{1}{6} H_{m n p} \mathrm{~d} y^{m} \wedge \mathrm{d} y^{n} \wedge \mathrm{d} y^{p}
$$

whose equation of motion give us a dual 6 -form $C_{6}$ such that

$$
\mathrm{d} C_{6}=*_{10+1} \mathrm{~d} C_{3}=e^{-2 \Phi} *_{9+1} H,
$$

where the second Hodge-star operation is with respect to the string metric as before. Of course, we may now identify $d B_{6}=d C_{6}$. Instead of setting $B_{6}=C_{6}$, let us leave the additive gauge freedom in definition of $C_{6}$ for a while.

According to Ref. [38, an M5-brane with 4 translational symmetries along $x^{\mu}$ may be calibrated as follows. The energy density functional per unit 3-volume is composed of two pieces. The first is the warped volume density

$$
\int_{\Sigma} \sqrt{-\operatorname{Det}\left(e^{-2 \Phi / 3} \eta\right)} \cdot \sqrt{\operatorname{Det}\left(e^{-2 \Phi / 3} h\right)},
$$

with the induced metric $h$ from $g$ on the embedding surface $\Sigma$, which is actually

$$
\int_{\Sigma} e^{-2 \Phi} \sqrt{\operatorname{Det}(h)}
$$


The second piece is the Coulomb energy density, which goes as

$$
\left.-\int \Sigma^{*}\left(\operatorname{vol}_{3+1}^{\eta}\right\lrcorner C_{6}\right) .
$$

The sum of these two terms are total energy density of the configuration

$$
\left.\mathcal{E}^{\prime}=\int_{\Sigma} e^{-2 \Phi} \sqrt{\operatorname{Det}(h)}-\int \Sigma^{*}\left(\operatorname{vol}_{3+1}^{\eta}\right\lrcorner C_{6}\right) .
$$

For calibration, there exists a closed 2-form $K$ such that

$$
\mathcal{E}^{\prime} \geq \int_{\Sigma} K
$$

is saturated precisely for the supersymmetrically wrapped branes. The deformation of the surface $\Sigma \rightarrow \Sigma^{\prime}$ would change the energy $\mathcal{E}^{\prime}$ while

$$
\int_{\Sigma^{\prime}} K-\int_{\Sigma} K=\int_{V} \mathrm{~d} K=0
$$

with the interpolating volume $V$ whose two boundaries are $\Sigma$ and $\Sigma^{\prime}$. Thus, such a $K$, to be found via supersymmetry conditions, will provide the absolute minimum energy to be saturated.

The closed form $K$ is found as follows. One first finds a covariantly constant spinor, $\epsilon$, which is responsible for the supersymmetry of the background geometry. The supercharge $\mathcal{Q}(\epsilon)$ associated with $\epsilon$ will have the property

$$
2 \mathcal{Q}(\epsilon)^{2}=\mathcal{E}^{\prime}-\int_{\Sigma} K
$$

ensuring the generalized calibration. The 2 -form $K$ is found to be

$$
\left.K=-\operatorname{vol}_{3+1}^{\eta}\right\lrcorner C_{6}+\bar{\epsilon} \Gamma \epsilon \sqrt{-\operatorname{Det}\left(e^{-2 \Phi / 3} \eta\right)},
$$

where $\Gamma$ is the pull-back of $\Gamma_{A B} \mathrm{~d} X^{A} \mathrm{~d} X^{B}$ to $\Sigma$. $\Gamma$ differs from its counterpart in the heterotic string theory, call it $\gamma$, by an overall factor of $e^{-2 \Phi / 3}$. Then the final form of $K$ is

$$
\left.K=-\operatorname{vol}_{3+1}^{\eta}\right\lrcorner C_{6}+e^{-2 \Phi} \Sigma^{*}\left(\bar{\epsilon} \gamma_{m n} \epsilon \mathrm{d} x^{m} \mathrm{~d} x^{n}\right) .
$$

The bulk 2-form in the latter term, we realize to be precisely the fundamental 2-form $J$ of the internal dimension, so we have

$$
\left.K=-\operatorname{vol}_{3+1}^{\eta}\right\lrcorner C_{6}+e^{-2 \Phi} \Sigma^{*}(J) .
$$


$K$ should be closed, $\mathrm{d} K=0$, if we have supersymmetry in the bulk, and indeed this follows from one of our supersymmetric equation for any heterotic flux background, $e^{-2 \Phi} * H=\mathrm{d}\left(e^{-2 \Phi} J\right)$. Finally the two $C_{6}$ pieces in $\mathcal{E}^{\prime}$ and $K$ cancel each other, and

$$
\mathcal{E}=\mathcal{E}^{\prime}-K=0
$$

is the generalized calibration condition, regardless of the gauge choice for $C_{6}$, as we promised above.

\section{A Smooth Compactification and Calibration}

A nonsingular flux compactification of the heterotic string theory was recently found by Fu and Yau [27, which was further elaborated on by Becker et.al. [28] The string theory on this background is believed to be U-dual to an orientifold limit of $K 3 \times K 3$ compactification of F-theory with fluxes turned on. One can describe the geometry as a $T^{2}$ fibred over a conformally rescaled $K 3$, where the metric and the fundamental 2-form are

$$
g=e^{2 \Phi} g_{K 3}+|\theta|^{2},
$$

and

$$
J=e^{2 \Phi} J_{K 3}+\frac{i}{2} \theta \wedge \bar{\theta},
$$

with a holomorphic 1 -form $\theta$. The dilaton is a function on $K 3$ only. Locally $\theta$ should have the form

$$
\theta=\mathrm{d} z+\alpha,
$$

with ill-defined 1-form $\alpha$ on K3, where $z$ is the holomorphic coordinate on the fibre $T^{2}$. Well-defined $T^{2}$ bundle over $K 3$ requires that we have integral Chern classes, which imposes that "real" and "imaginary" parts of the 2-form

$$
\omega=\frac{\mathrm{d} \theta}{2 \pi \sqrt{\alpha^{\prime}}},
$$

belong to integral cohomology of $K 3$. Since we must have

$$
0=\mathrm{d}\left(e^{-2 \Phi} J \wedge J\right) \quad \Rightarrow \quad 0=i J_{K 3} \wedge(\omega \wedge \bar{\theta}-\theta \wedge \bar{\omega}),
$$

we must also require the primitivity of $\omega$ in $K 3$.

$$
\omega \wedge J_{K 3}=0 .
$$


These exhaust constraints on the geometry except for determination of the dilaton on $K 3$ and supersymmetry condition on the gauge bundle. Supersymmetry condition on the gauge bundle is the familiar one. Namely, the field strength $F$ should be of type $(1,1)$ and primitive with respect to $J$.

This ansatz solves all supersymmetry relationships except for actual form of $\Phi$, where all functional information of the solution is encoded. The authors of Ref. [27] choose to extract the equation for $\Phi$ from the Bianchi identity,

$$
\mathrm{d} H=\frac{\alpha^{\prime}}{4}\left[\operatorname{tr}\left(R^{\prime} \wedge R^{\prime}\right)-\operatorname{tr}(F \wedge F)\right]
$$

where they take the curvature 2 -form $R^{\prime}$ to be that of the so-called Hermitian connection. This choice involves a torsion which is not completely anti-symmetric and thus cannot be of the form, $\sim a H$. Nevertheless, the size of this torsion is the same order as $H$, and this choice represents a correction term of order $\sim\left(\alpha^{\prime}\right)^{2}$ in this equation. ${ }^{\ddagger}$ Also this has the advantage that the right hand side is $(2,2)$ Hodge type, allowing the equation self-consistent without a further $\alpha^{\prime}$ truncation. We will come back to this anomaly equation later on to discuss a crucial tadpole condition.

Before proceeding further, let us note that the sizes of the base and of the fibre are free and also that the zero mode of dilaton, $\Phi_{0}$, is free. A better way to write the ansatz is,

$$
g=e^{2\left(\Phi-\Phi_{0}\right)} R_{B}^{2} \hat{g}_{K 3}+l_{F}^{2}|\theta|^{2},
$$

and

$$
J=e^{2\left(\Phi-\Phi_{0}\right)} R_{B}^{2} \hat{J}_{K 3}+\frac{i l_{F}^{2}}{2} \theta \wedge \bar{\theta}
$$

where the volume of $K 3$ in terms of $\hat{g}_{K 3}$ is normalized to unit. The linear size of the fibre is $2 \pi l_{F} \sqrt{\alpha^{\prime}}$ and the linear size of the base is $R_{B}$, both of which are free parameters of the solution. The solution would be trustworthy when $R_{B}^{2} / \alpha^{\prime} \gg 1$ and $l_{F} \gg 1$. In the following discussions, however, $l_{F}$ factor either cancels out or appears as an overall coefficient while $e^{-\Phi_{0}} R_{B}$ can be absorbed into the definition of the $K 3$ metric as $g_{K 3}=e^{-2 \Phi_{0}} R_{B}^{2} \hat{g}_{K 3}$ and similarly of the Kähler form. We will suppress these factors, with the understanding that they can be restored easily and also that their large sizes are important for our discussions.

\footnotetext{
${ }^{\ddagger}$ For smooth and large flux compactification, it turns out that the size of $H^{2}$ has to be of order $\alpha^{\prime}$. See Ref. 25] for more detailed explanation. This fact also holds in this solution, naturally.
} 
This heterotic flux compactification is deemed to be U-dual to a well-known Ftheory compactification on $K 3 \times K 3$, or more precisely an orientifold limit thereof, provided that we add some additional RR and NS-NS fluxes, $F_{3}$ and $H_{3}$, on the latter [28, 15]. As was shown by Sen [39], F-theory on $K 3 \times K 3$ [40] is a generalization of the orientifold $T^{2} / Z_{2}$ times $K 3$ of IIB theory, whereby we move around the D7-branes located at the four tips of $T^{2} / Z_{2}$. In the orientifold limit, each tip represents one $O 7^{-}$ plane, 4 pairs of $D 7$ branes, and the associated $S O(8)$ gauge groups on D7's.

The duality chasing starts with T-dualization of the $T^{2} / Z_{2}$. This will change $O 7^{-}$ and $D 7$ 's into $O 9^{-}$and $D 9$ 's, and the orbifold is now resolved to regular $T^{2}$. However, the presence of NS-NS flux $H_{3}$ means that certain off-diagonal value of metric will be generated [4], so the geometry on this type I side will be such that $T^{2}$ has nontrivial mixing with $K 3$ in the metric. Then, we can switch over to the heterotic side by taking S-duality [42, 43], whereby mapping $F_{3}$ to $H$.

In this note, we are mostly interested in understanding possible brane configurations on the two sides and comparing their properties, in part because this will strengthen this duality conjecture and also in part this could diversify possible models based on such compactification. As we noted above, the IIB background comes with D7 branes as part of the geometric data. On top of this, we can think of two more classes of D-brane configurations with supersymmetry. One class consists of D3 branes transverse to the compact directions. The other is D7 branes intersecting with the indigenous D7 branes. The latter wraps $K 3$ while the former wraps $T^{2} / Z_{2}$ and a 2 -cycle on $K 3$. The latter would be supersymmetric only under a tight restriction on bulk complex moduli, unlike the former. We will come back to moduli of these D-branes in a later section.

Interestingly, both classes of these D-branes map to D5's in type I, and then to fivebranes upon S-duality to the heterotic side. D3 can be seen to correspond to fivebrane wrapping the fibre $T^{2}$, while D7's wraps 2-cycles in the base K3. For the rest of this note, we will consider issues related to these calibrated fivebranes on the heterotic side. A simplifying fact is that the presence of $e^{2 \Phi}$ factor on the conformal rescaling of $K 3$, so that the Coulomb energy density has a simple form,

$$
\int \Sigma^{*}\left(e^{-2 \Phi} J\right)=\int \Sigma^{*}\left(J_{K 3}\right)+\frac{i}{2} \int \Sigma^{*}\left(e^{-2 \Phi} \theta \wedge \bar{\theta}\right) .
$$


Since $\mathrm{d} J_{K 3}=0$, the first piece in the right-hand-side is topological

$$
\delta \int \Sigma^{*}\left(J_{K 3}\right)=0,
$$

so the problem of minimizing the energy functional becomes that of saturating the bound

$$
\mathcal{E}^{\prime}=\int_{\Sigma} e^{-2 \Phi} \operatorname{vol}_{\Sigma}-\frac{i}{2} \int \Sigma^{*}\left(e^{-2 \Phi} \theta \wedge \bar{\theta}\right) \geq \int \Sigma^{*}\left(J_{K 3}\right) .
$$

In terms of the generalized calibration presented in the previous section, this split corresponds to the choice of gauge for $C_{6}$,

$$
C_{6}=\frac{i}{2} e^{-2 \Phi} \theta \wedge \bar{\theta} \wedge v o l_{3+1}^{\eta},
$$

forcing

$$
K=J_{K 3},
$$

which is indeed a closed 2-form. In the large volume limit, $\mathcal{E}^{\prime}$ is a well-normalized measure of the energy density associated with the fivebrane.

The configuration $\Sigma$ saturating the lower bound $\mathcal{E}^{\prime}(\Sigma)=\int \Sigma^{*}(K)$ or equivalently $\mathcal{E}=0$ satisfies the local condition

$$
\operatorname{vol}_{\Sigma}=\Sigma^{*}(J) .
$$

Since $J$ is a Hermitian $(1,1)$ form on $\mathcal{M}_{6}$, this condition is satisfied if the embedding of $\Sigma$ into $\mathcal{M}_{6}$ is holomorphic. In the background geometry described in section 3, two independent tangent vectors of $\Sigma$ pushed forward into the bulk may be written as

$$
\xi+a \partial_{z}, \quad \bar{\xi}+\bar{a} \partial_{\bar{z}}
$$

with suitable coefficient $a$. Here $\xi$ is a tangent vector in $K 3$, which is $(1,0)$ with the complex structure $J_{K 3}$. Since $\mathcal{M}_{6}$ is a bundle over the base $K 3$, let us call the bundle projection map, $\pi$,

$$
\pi: \mathcal{M}_{6} \rightarrow K 3 .
$$

One can see that the image $\pi(\Sigma)(\subset K 3)$ of the holomorphic embedding $\Sigma$ is also holomorphic, with tangent vectors $\xi$ and $\bar{\xi}$. The holomorphic surface $\pi(\Sigma)$ determines the value of the integral at the right hand side of (4.12). Thus, we can think of two distinct classes of solutions, depending on whether the pull-back of $J_{K 3}$ integrates to zero or not. 


\section{Fivebranes on $T^{2}$, Cyclic Homotopy, a Tadpole, and U-Dual D3 Branes}

We first consider the case

$$
\int \Sigma^{*}\left(J_{K 3}\right)=\int(\pi(\Sigma))^{*}\left(J_{K 3}\right)=0 .
$$

A holomorphic embedding $\pi(\Sigma)$ in $K 3$ with vanishing integral (5.1) is necessarily point-like. Therefore, $\Sigma$ can wrap only the $T^{2}$ fibre and is localized at the point $\pi(\Sigma)$ in the base. We may take the complex coordinate $\zeta$ on the internal part of the worldvolume embedded as

$$
z=\zeta
$$

where $z$ is the complex coordinate of the fiber introduced in section 2, with identifications $\zeta \sim \zeta+2 \pi m \sqrt{\alpha^{\prime}}$ and $\zeta \sim \zeta+2 \pi n i \sqrt{\alpha^{\prime}}$ ( $m, n$ are integers) since $\Sigma$ is topologically a torus now. Note that the saturation (5.1) of $\mathcal{E}(\Sigma) \geq 0$,

$$
\Sigma^{*}\left(e^{-2 \Phi} J\right)=\frac{i}{2} \Sigma^{*}\left(e^{-2 \Phi} d z \wedge d \bar{z}\right)=e^{-2 \Phi} \operatorname{vol}_{\Sigma}
$$

occurs automatically for a completely vertical configuration. With this, we have $\mathcal{E}^{\prime}(\Sigma)=0$.

An interesting fact about the fibre $T^{2}$ is that it does not correspond to an element of the homology group with real coefficients. The effect of twisting due to $\omega_{i}$ is that the two circles of $T^{2}$ can become a contractible loop. As a toy example, take an $S^{1}$ fibred over $S^{2}$ via Hopf fibration. The metric goes as

$$
\mathrm{d} s_{S^{2}}^{2}+(\mathrm{d} \psi+k \cos \theta \mathrm{d} \phi)^{2}
$$

with the Hopf number $k$. The resulting topology of the bundle is $S^{3} / Z_{k}$ and its first homotopy group is

$$
\pi_{1}=Z_{k}
$$

A loop that winds around the fibre $S^{1} k$-times becomes contractible. The twisting in $\mathcal{M}_{6}$ is essentially the same type of fibration, except that we now have a pair of $S^{1}$ 's and that the two-dimensional bases are replaced by 2-cycles in $K 3$. Because of this, a fivebrane wrapping $T^{2}(m, n)$-times will be homotopically trivial when $m$ or $n$ 
equals some integer $k$ whose precise value is determined by the bundle $\mathcal{M}_{6}$. Because a fivebrane can unwrap when either of the circle becomes contractible, there are in general at least two such integers $k_{1}$ and $k_{2}$, associated with the two circles of $T^{2}$ fibre, so that we have in general processes that shifts

$$
\Delta N_{\text {fivebranes on } T^{2}}=a_{1} k_{1}+a_{2} k_{2}
$$

for integers $a_{1}$ and $a_{2}$.

A simple generalization of this toy model is to consider $S^{1}$ fibred over $\left(S^{2}\right)^{K}$, with the metric being

$$
\sum_{p=1}^{K} \mathrm{~d} s_{S_{p}^{2}}^{2}+\left(\mathrm{d} \psi+\sum_{p} m_{p} \cos \theta_{p} \mathrm{~d} \phi_{p}\right)^{2}
$$

in which case the homotopy group would be $Z_{k}$ with $k$ being the greatest common divisor of $\left\{m_{p}\right\}$. Although in our case the 1-forms that enter the last terms are associated with 2 -cycles in a single $K 3$, this still suggests that $k_{1,2}$ above should be determined similarly by what integral linear combination of the generators $H^{2}(K 3, Z)$ is used for $\omega_{1,2}$.

However, this does not mean that the dominant unwrapping processes are the ones that shift the winding number by $k_{1}$ and $k_{2}$. Depending on precise geometry, some linear combination like $2 k_{1}+5 k_{2}$ could prove to be the easiest path. Homotopy does not know anything about the dynamics. In terms of the toy model geometry, the "easiest" path would correspond to shift of the winding number by $a_{1} k_{1}+a_{2} k_{2}=m_{p}$ if $p$-th $S^{2}$ happens to be much smaller than all the others.

In any case, with such a cyclic nature of the winding number, our finding that fivebrane wrapping $T^{2}$ is stable and supersymmetric, may sound strange. Wrapping it $k$-times will result in homotopically trivial configuration which can be unraveled and made to contract to nothing. In fact, depending on details of the metric, it may even be possible to deform $\Sigma$ away from this vertical configuration and reduce its area. The point is that such a deformation is always accompanied by a cost in the Coulomb energy,

$$
\Delta E_{\text {electric }}=-\int_{\Sigma^{\prime}} \frac{i}{2} e^{-2 \Phi} \theta \wedge \bar{\theta}+\int_{\Sigma} \frac{i}{2} e^{-2 \Phi} \theta \wedge \bar{\theta}
$$

and that this cost always override, if any, the energy gain from the reduction of the 
area when the fivebrane is deformed away from $T^{2}$. It is the magnetic Coulomb energy that protects such nontopological configurations. ${ }^{\S}$

We just argued that the winding number of fivebrane over the fibre $T^{2}$ is additive only modulo some integers $k_{i}$ 's. Consistent with this fact is that there is no element of $H^{2}\left(\mathcal{M}_{6}, R\right)$ which is Poincare-dual to the $T^{2}$ fibre [44, 28. This non-extensive or cyclic nature of $T^{2}$ winding number raises a number of interesting questions.

First, recall that fivebranes contribute to the tadpole condition for $H$ since it is a magnetic source. The $T^{2}$ winding number will act as a magnetic source to $H$ field along $K 3$. How is it possible that such a tadpole source can be unwrapped and disappear? The resolution to this quandary comes from the fact that $K 3$ is not a cycle in the manifold $\mathcal{M}_{6}$. The fivebrane in question wraps the fibre $T^{2}$, and therefore can contribute to a tadpole condition along the base $K 3$. Normally this tadpole condition would arise from integrating $\mathrm{d} H$ equation over a homology cycle representing $K 3$. However, there is no such cycle as long as the $T^{2}$ bundle is nontrivial. Of course, in the present geometry, we can unwrap the fivebrane on fibre precisely when the $T^{2}$ bundle is nontrivial.

Instead, the relevant tadpole condition arises from integration of $J \wedge \mathrm{d} H=\cdots$ over the entire manifold,

$$
\int_{\mathcal{M}_{6}} J \wedge \mathrm{d} H=\frac{\alpha^{\prime}}{4}\left[\int_{\mathcal{M}_{6}} J \wedge\left(\operatorname{tr} R^{\prime} \wedge R^{\prime}-\operatorname{tr} F \wedge F-16 \pi^{2} \delta_{\text {fivebrane }}\right)\right] .
$$

Note that the left hand side does not vanish. Instead we have

$$
\int_{\mathcal{M}_{6}} J \wedge \mathrm{d} H=4 \pi^{2} \alpha^{\prime} \int_{K 3}|\omega|^{2}
$$

where the contraction of $\omega$ is taken with respect to the Calabi-Yau metric $g_{K 3}$ without the dilaton factor. The right hand side can also be computed, and we have the following tadpole condition

$$
N_{\text {fivebrane on } T^{2}}+\int_{K 3}|\omega|^{2}=\frac{1}{16 \pi^{2}} \int_{K 3} \operatorname{tr} R_{K 3} \wedge R_{K 3}-\operatorname{tr} F \wedge F .
$$

\footnotetext{
$\S$ While the energy density $\mathcal{E}^{\prime}$ is such that the fivebrane winding the $T^{2}$ fibre has the same energy as the trivial configuration, one should not take this mean that the $T^{2}$ wrapped fivebrane is tensionless. The coupling to the Coulomb field does not enter the dynamics of the general worldvolume excitations.
} 
The right-hand-side is

$$
-\frac{1}{2} p_{1}(K 3)+\frac{1}{2} p_{1}(\mathcal{F})
$$

in terms of the Pontryagin class $p_{1}$. We have $-p_{1}(K 3) / 2=24$ while $-p_{1}(\mathcal{F}) / 2$ is a nonnegative integer. Note that this equation does reflect the expectation that the fivebrane number is interchangeable with the topological number on the right hand side.

Now consider the process of unwrapping a fivebrane on $T^{2}$ with all backreaction taken into account. This process will necessarily involve a fivebrane source which is spread over the base $K 3$ as well as over $T^{2}$. The backreaction of the metric and the torsion is then such that this fibre-bundle form of the geometry is completely destroyed throughout the middle step. Since we are not resolving the fivebrane into the gauge bundle, neither of the Pontryagin numbers will change upon completion of this unwrapping process, leading to our quandary.

However, the second term on the left, which has something to do with the winding of $T^{2}$ fibre can easily change. The initial and the final configurations are $T^{2}$ bundles over $K 3$, but the interpolating geometries cannot be one. This deformation of the bundle structure should absorb the difference in $N_{\text {fivebrane on } T^{2}}$ and encode it in the integer shift of the integral of $|\omega|^{2}$. This could occur via a shift of the integral cohomology associated with $\omega_{1,2}$, or due to change of metric on the base $K 3$. In the former case, another $T^{2}$, which is different from the initial $T^{2}$ fibre, emerges as the fibre at the end of the process. In the latter case, the cohomology class of $\omega_{1,2}$ remains unchanged but their split into self-dual and anti-self-dual part can change. Because the intersection pairing (relevant to cohomology class) and the norm differ by a sign in anti-self-dual part of $H^{2}(K 3)$, this can also shift the integrated value of $|\omega|^{2}$.

This does not mean that a homotopically trivial fivebrane can decay to nothing. The winding configuration represents a supersymmetric vacuum in $3+1$ dimensional effective theory, and thus cannot decay to another supersymmetric vacua. It only means that we have two (or more) degenerate configurations with different $T^{2}$ winding numbers of the fivebrane. In particular, there should be supersymmetric domain wall configurations separating these degenerate vacua from one another. It is not difficult to see that the domain walls themselves should be represented by fivebranes wrapping a 2-cycle in $K 3$ and one of the two circles in $T^{2}$. Following how configuration changes 
as we move from the side with winding to the other side, one should see the fivebranes gradually unwinding as a function of the transverse coordinate.

So far, we addressed various issues entirely within the heterotic theory. Here we would like to close the section by studying how this unusual nature of $T^{2}$ wrapped fivebranes manifest itself in the U-dual picture. As we noted earlier, these fivebranes map to D3 branes in IIB orientifold. Seemingly, D3 branes carry an integer conserved charge, and we have a potential conflict.

This can be made more dramatic by replacing D3 by anti-D3. In the heterotic side, one wraps the fivebrane over $T^{2}$ with opposite orientation. Since this breaks supersymmetry, the false vacuum will try to decay into a supersymmetric one taking away $k$ unit of anti-fivebranes. Anti-fivebranes on $T^{2}$ have an energy function which is just twice the tension and would favor being unwrapped out of $T^{2}$. Without topology protecting them, a $k$ number of anti-fivebranes on $T^{2}$ can then disappear either classically or by tunneling. Can anti-D3's also disappear in some quantized unit on IIB side? Although we just phrased the question in terms of anti-D3's to make the possible conflict more obvious, the same sort of question exists for D3's as well. In term of the latter, the question is whether there are supersymmetric domain wall configurations with different number of D3 branes on the two sides.

On IIB side, D3 tadpole condition involves a flux contribution so that we have

$$
0=\int F_{3} \wedge H_{3}+N_{D 3}
$$

where appropriate normalization constants are understood. On the other hand, $F_{3}$ and $H_{3}$ fluxes can jump in quantized unit across a domain wall formed by a D5-brane or by a NS5-brane wrapping a 3-cycle [45. The tadpole must be preserved no matter what, and $N_{D 3}$ will thus jump across such a domain wall.

When one side of this domain wall contains anti-D3 branes, the domain wall can form a bubble, inducing decay of a false vacuum with anti-D3 into another false vacuum with lesser number of anti-D3 or into a true vacuum with no anti-D3 branes. The precise unit in which $N_{D 3}$ jumps depends on what are initial fluxes in $F_{3}$ and $H_{3}$, and which 3-cycle is used for wrapping NS5 or D5 branes to form the domain wall. If $H_{3}$ flux is shifted by unit via a domain wall from NS5 wrapping a cycle $A$, $N_{D 3}$ will shift by an integer

$$
\oint_{B} F_{3}
$$


where $B$ is the dual 3-cycle of $A$, and vice versa. Set of these integers, that is, possible shifts of $N_{D 3}$ via such domain walls, should match precisely the allowed linear combinations of the two integers $k_{1,2}$ 's we saw in the heterotic side, if the Uduality holds in the presence of the flux. As in the heterotic case, if one side contains anti-D3 branes, breaking supersymmetry, the wrapped D5 or NS5 branes will instead appear as an expanding bubble in $3+1$ dimensions, removing anti-D3 branes in these quantized units.

Finally, it is not entirely obvious why the unwrapping of anti-fivebranes has to be via tunneling on the heterotic side. Of course the U-dual map involves a strong-weak coupling duality, so we cannot compare directly. Still, ignoring quantum issues, it looks likely that, even in the heterotic side, the decay of anti-fivebrane on $T^{2}$ would occur via tunneling. Taking T-dual of a large IIB orientifold along the $T^{2} / Z_{2}$, the base manifold remains large while the fibre may not. An important fact is that the ratio of the size of the base and that of the fibre could be a large number, and this is unaffected by the final S-duality into the heterotic side. Furthermore, antifivebrane on $T^{2}$ will settle down to a point where $e^{-2 \Phi}$ is minimized along $K 3$, where the conformally rescaled size of $K 3$ is maximal. These two act together to force the unwrapping process to be a tunneling process, if the fibre size is much smaller. Unwinding necessarily involves fivebrane wandering into $K 3$, which could come at the cost of much larger area. Recall that for anti-fivebrane the net energy is bounded below by the tension energy due to the area, since the wrong sign of the Coulombic energy term adds rather than subtract. Also, being at local minimum of $e^{-2 \Phi}$ implies that unwrapping will cost even more because the configuration must move away from the local minimum of this factor also.

There are still more details of this matching between $T^{2}$ wrapping fivebranes and D3 branes that must be checked. In particular, it remains a challenging problem to match of all possible tunneling processes on F/IIB side which changes the number of D3 branes against those in the heterotic side which changes the $T^{2}$ winding number. Equivalently, one would like to match all possible domains walls of the two sides. However, a precise matching of this kind would go a long way in establishing the duality map we have been using and should be a worthwhile exercise. 


\section{Fivebranes on 2-Cycles in $K 3$}

Next, we consider the case

$$
\int \Sigma^{*}\left(J_{K 3}\right) \neq 0
$$

In this case, $\pi(\Sigma)$ spans a two dimensional surface, and represents a nontrivial element in the second homology $H_{2}(K 3)$ group. To saturate the calibration bound condition, $\pi(\Sigma)$ must be a holomorphic embedding in $K 3$, which means that the pull-back of the holomorphic $(2,0)$-form of $K 3$ vanishes

$$
0=\pi(\Sigma)^{*}\left(\Omega_{K 3}^{(2,0)}\right)
$$

Once we find such a holomorphic embedding $\pi(\Sigma)$, we should search for a uplift it to the total manifold $\mathcal{M}_{6}$.

This uplifting is not possible unless there is a global section. That is, this last step is possible only if the restriction of the bundle over $\pi(\Sigma)$ is a trivial bundle. This in turns requires that the Chern class of the bundle integrates to zero over this surface, so that we must have

$$
\int_{\Sigma} d \alpha=0 \rightarrow \int_{\Sigma} \omega_{1}=\int_{\Sigma} \omega_{2}=0
$$

as well. Once this holds, it comes down to what kind of uplifting is available, which will depend on topology of $\pi(\Sigma)$.

Because the restricted bundle $\pi^{-1}(\pi(\Sigma)) \subset \mathcal{M}_{6}$ is trivial, we can also talk about $T^{2}$-winding number of this uplifting. That is, $\Sigma$ may wind around the fibre once or more, depending on the topology of $\pi(\Sigma)$, and this would provide additional quantum number associated with $\Sigma$. Note that this $T^{2}$ winding number does not contribute to $\mathcal{E}^{\prime}$ since the contribution to the area is canceled by the Coulomb energy point-wise. Thus, the uplifting of $\pi(\Sigma)$ with additional $T^{2}$ winding number represents a threshold bound state of $\Sigma$ without the $T^{2}$ winding number and a number of the $T^{2}$ wrapping fivebrane.

While the above more or less characterize solutions to the supersymmetry conditions, we can do things more explicitly thanks to the well-known description of the homology of $K 3$. We can translate all of above as a set of restrictions on various intersection numbers. Let us call the generators of this homology $\Sigma_{I}(I=1, \cdots, 22)$, 
whose intersection numbers are given by the matrix

$$
C_{I J}=\left(\begin{array}{ccccc}
-E_{8} & & & & \\
& -E_{8} & & & \\
& & U & & \\
& & & U & \\
& & & & U
\end{array}\right) \quad, \quad U=\left(\begin{array}{ll}
0 & 1 \\
1 & 0
\end{array}\right),
$$

where $E_{8}$ denotes the $8 \times 8$ Cartan matrix of the $E_{8}$ Lie algebra. We expand

$$
[\pi(\Sigma)]=\sum_{I=1}^{22} n^{I}\left[\Sigma_{I}\right]
$$

where $n^{I}$ are integers. The 2 -forms $\eta_{I}$ Poincare-dual to $\Sigma_{I}$ are defined by the relation

$$
\int_{K 3} \eta_{I} \wedge v=\int_{\Sigma_{I}} v
$$

for any closed 2-form $v$.

$J_{K 3}$ is covariantly constant in terms of $K 3$ metric, and can be expanded as

$$
J_{K 3}=\sum_{I=1}^{22} h^{I} \eta_{I}
$$

with real $h^{I}$. K3 is Calabi-Yau, or equivalently, hyperKähler, and has a holomorphic 2 -form $\Omega_{K 3}^{(2,0)}$, which can also be represented as a linear combination of $\eta$ 's,

$$
\Omega_{K 3}^{(2,0)}=\sum_{I=1}^{22} t^{I} \eta_{I}
$$

The pull-back of $\Omega^{(2,0)}$ must vanish on $\pi(\Sigma)$, so we have the first necessary condition for calibrated $\pi(\Sigma)$

$$
\int_{\pi(\Sigma)} \Omega_{K 3}^{(2,0)}=\sum_{I J} C_{I J} n^{I} t^{J}=0 .
$$

Furthermore, the surface should have the right orientation to have positive integral of $J_{K 3}$ which demands that

$$
\int_{\Sigma} \Sigma^{*}\left(J_{K 3}\right)=n_{I} h^{J} \int_{\Sigma_{I}} \eta_{J}=C_{I J} n^{I} h^{J}>0 .
$$

Obviously at least one of $n^{I}$ should be nonvanishing. 
Given such a holomorphic embedding $\pi(\Sigma)$, there is well-known counting of its moduli in $K 3$. Thanks to $\Omega^{(2,0)}$, counting of deformation become counting of $H^{1}(\Sigma)$ [46], which is in turn related to the Euler number as

$$
\operatorname{dim} H^{1}(\Sigma)=2-\chi=2 g
$$

There are in general $2 g$ real deformation moduli for genus $g$ surface $\pi(\Sigma)$. In terms of the above decompositions into integral cohomology, there is an alternate formula for this number, which goes as

$$
2 g \stackrel{\star}{=} 2-\int_{\Sigma} c_{1}\left(T_{\pi(\Sigma)}\right) \stackrel{\checkmark}{=} 2+\int_{\Sigma} c_{1}\left(N_{\pi(\Sigma)}\right)=2+\int_{K 3} \sigma \wedge \sigma=2+C_{I J} n^{I} n^{J}
$$

where at $\checkmark$ we used the fact $0=c_{1}(K 3)=c_{1}(T)+c_{1}(N)$, and at $\star$ we assumed that the surface is connected and smooth. The $\sigma$ in the integrand denotes the Poincare dual of the 2-cycle $\pi(\Sigma), \sigma=\sum_{I} n^{I} \eta_{I}$. Existence of such a holomorphic embedding implies that the deformation parameter is nonnegative, so we arrive at

$$
C_{I J} n^{I} n^{J} \geq-2
$$

which is the 3rd necessary condition.

So far we considered the implication of having nontrivial holomorphic image $\pi(\Sigma)$ in the $K 3$ base. As we saw above, uplifting this to a holomorphic surface in $\mathcal{M}_{6}$ requires $\pi(\Sigma)$ be orthogonal to $\omega_{1,2}$ under the intersection pairing. Writing

$$
\omega_{i}=\sum_{I=1}^{22} l_{i}^{I} \eta_{I} \quad\left(i=1,2, \quad l_{i}^{I} \text { are integers }\right)
$$

we have the 4th necessary condition (6.3) as

$$
C_{I J} n^{I} l_{i}^{J}=0
$$

When this holds a global section exists, and we could uplift $\pi(\Sigma)$ into $\mathcal{M}_{6}$.

Summarizing, we have several necessary conditions among the homology cycle $\pi(\Sigma)=n_{I}\left[\Sigma_{I}\right], J_{K 3}=h^{I} \eta_{I}, \Omega_{K 3}^{(2,0)}=t^{I} \eta_{I}$, and $\omega_{i}=l_{i}^{I} \eta_{I}$

- $C_{I J} n^{I} t^{J}=0$

- $C_{I J} n^{I} h^{J}>0$ 
- $C_{I J} n^{I} n^{J} \geq-2$

- $C_{I J} n^{I} l_{i}^{J}=0$

in addition to the bulk supersymmetry conditions

- $C_{I J} h^{I} t^{J}=0$

- $C_{I J} t^{I} t^{J}=0$

- $C_{I J} h^{I} l_{i}^{J}=0$

all of which must hold in order for the supersymmetric $\Sigma$ to exists.

There could be at most 20 independent integer vectors $n^{I}$ 's which solve the these equations. The precise number of which depends on whether $\omega_{i}$ 's are entirely along $(2,0)$ or has component in $H^{(1,1)}$. Even for those solution that solves all of above requirement, many of them cannot be realized as a single holomorphic surface of the right orientation. As a crude example, working with a 2 dimensional subspace, we take $\omega_{1}=(1, \pm 1), \omega_{2}=(0,0)$ with

$$
C_{I J} \sim\left(\begin{array}{ll}
2 & 0 \\
0 & 2
\end{array}\right)
$$

The solution $u^{I}$ satisfying $C_{I J} \omega_{i}^{I} n^{J}=0$ is $n^{I}=(1, \mp 1)$ in each case. The upper sign should be forbidden since it has wrong orientation.

Another set of interesting example where solution to the above algebraic equations does not guarantee actual holomorphic surface can be found when we confine ourselves to generators of one of $E_{8}$ factor in the $H_{2}(K 3)$. Here we expect only those surfaces which actually exists as supersymmetric state are those corresponding to a root of $E_{8}$. Because $-C_{I J} n^{I} n^{J}$ measures the length squared of the corresponding root, all 2cycles of this kind are spheres with $g=0$. Other combinations such as $\alpha+2 \beta$, where $\alpha$ and $\beta$ are pair of distinct roots, may be arranged to solve all of above constraints but cannot corresponds to an irreducible, smooth, connected, and holomorphic surface.

Reversely, the existence of such holomorphic $\pi(\Sigma)$ imposes constraints on the base $K 3$ manifold. Each wrapped 2-cycle of this kind must belong to the set

$$
\operatorname{Pic}(K 3) \equiv H^{2}(K 3, \mathbb{Z}) \cap H^{1,1}(K 3)
$$


which is called the Picard lattice. The Picard lattice is null for generic $K 3$, while the rank of this lattice can be as larger as 20 by adjusting $\Omega_{K 3}^{(2,0)}$ since $h^{1,1}=20$. When the rank is maximal the corresponding $K 3$ is called attractive [47]. This picks out $\Omega_{K 3}^{(2,0)}$ among discrete possibilities, so the remaining geometric moduli are all in the choice of $J_{K 3}$. Since $J_{K 3}$ should be orthogonal to $\Omega_{K 3}^{(2,0)}$, an attractive $K 3$ would have 20 moduli intact out of the original $58 K 3$ moduli.

However, this statement should be taken with a grain salt. Here we are pretending that we could ignore back reaction of the geometry to such wrapped fivebranes, and in particular are yet to take into account the modification of tadpole conditions due to these new sources. We believe consistent treatment of these effect will take us away from the $T^{2}$ fibre bundle over $K 3$, and will require much more elaborate geometry. The above statement of further fixing of bulk moduli should be taken verbatim in noncompact local models only, and for compact cases, backreaction of the geometry should be taken into account. On the other hand, having these extra fivebranes essentially generates further fluxes, either in the form of gauge bundle or $H$ itself. The idea of backreaction to additional fivebranes fixing more moduli must be robust. We wish to come back to this issue in a separate work.

\section{More on U-Dual and D-Branes}

U-dualizing to IIB orientifold $T^{2} / Z_{2} \times K 3$ involves an S-duality to type I and then T-duality on $T^{2}$. Following the usual rules, it is quite clear that these fivebranes on $T^{2}$ are U-dual to D3-branes in IIB side. Similarly fivebranes wrapping 2-cycles in $K 3$ are D7-branes, in IIB side, which wraps $T^{2} / Z_{2}$ as well as the same 2-cycles in $K 3$. Here let us address further issues related to this U-duality map between branes, such as the complete $3+1$ dimensional massless spectra of the two sides.

Before proceeding, we must first clarify which heterotic theory we are considering. Since we have a $T^{2}$ in the geometry, U-dualization can bring us to either of the pair of the heterotic theory with $E_{8} \times E_{8}$ or $S O(32)$, depending on whether we perform one more T-duality on one of the circle in the fibre or not. Also the supersymmetry requirement solved by the current solution is common to both theories, so it remains ambiguous which theory we are discussing. For us, the main difference is in fivebranes. Fivebranes in $E_{8} \times E_{8}$ theory are direct descendants of M5 branes, and are equipped 
with a tensor theory on worldvolume. Fivebranes wrapped on a circle maps under the T-dual map to fivebranes wrapped on a dual circle in $S O(32)$ heterotic theory, so the worldvolume theory on the latter must be a vector theory [48].

On the other hand, T-dual of a transverse fivebrane is instead a KK monopole solution. Thus, when we identify the fivebranes on a 2-cycle in $K 3$ as U-dual of D7 branes wrapping $T^{2} / Z_{2}$ and a 2-cycle in $K 3$, we are implicitly considering them in the heterotic theory which is S-dual to type I, without further T-dualization on the fibre, and this is $S O(32)$ heterotic theory. Thus, when we compare fivebranes in the heterotic side to D7 on IIB side, we are considering $S O(32)$ theory and fivebranes whose worldvolume theory is a vector theory.

With this said, the comparison of the spectra is well established in the absence of flux. Let us consider the low energy spectra of fivebranes wrapped on 2-cycle $\Sigma$ and those of D7 branes wrapped on $T^{2} / Z_{2}$ and $\Sigma$. Both carry a vector theory, and the only difference come from how $T^{2}$ worth of position moduli of the fivebrane arise from Wilson lines on D7 side. In addition, there are $g$ complex moduli with $g$ being the genus of $\Sigma$ and a vector on both sides. Similar consideration shows identical spectra for D3 and its U-dual. Generic D3 has 6 translational moduli and one 3+1 dimensional vector. These are also obvious from fivebranes on $T^{2}$. For each fivebrane on $T^{2}$, there are four translational degrees of freedom for a position on $K 3$. Four more bosonic massless degrees of freedom arise from the worldvolume vector multiplet (or the self-dual tensor multiplet) on the worldvolume, from two Wilson lines generating two scalars in addition to the vector field itself. " On IIB side, also without the fluxes, the same counting appears from the adjoint sector of D3 multiplet. The latter corresponds to a Coulomb phase massless degrees of freedom of a single $N=2 D=4$ $S U(2)$.

What may be less obvious is what happens to this correspondence when fluxes are turned on. For instance, it is well-known that D3's are attracted to D7's in the presences of NS-NS $B$-field along directions transverse to D3 and longitudinal to D7. This can be understood from the worldvolume theory of D3 as a Fayet-Illiopoulos D-term constants on D3, which lifts its Coulomb phase to have a finite and positive

IIf we were dealing with fivebranes from $E_{8} \times E_{8}$ side, the worldvolume has a tensor multiplet instead. But the same $3+1$ dimensional spectra arises since a $5+1$ dimensional supersymmetric tensor theory compactified on a circle gives the same field content as a $5+1$ dimensional supersymmetric vector theory. 
energy. This effect comes from anti-self-dual part of $B^{N S-N S}$ along $K 3$. D3 would then become a pair of non-commutative instantons in D7 gauge theory. U-dual map of this configuration is likely to be a gauge bundle over $K 3$ on the heterotic side, so this would imply that isolated fivebranes wrapping $T^{2}$ is not really there. Conversely, this lifting of those moduli of D3 along $T^{2} / Z_{2}$ would map on the heterotic side to massive Wilson lines on $T^{2}$, which seems very unlikely.

In this case, the problem is solved because of the detailed form of the NS-NS $B$-field. The twisting of the bundle, encoded in the holomorphic 1-form $\alpha$ on $K 3$, arises from T-dualization of NS-NS $B$ form on IIB side. The gauge choice which is convenient for the T-dualization is

$$
B^{N S-N S} \sim d f \wedge \alpha
$$

with $f$ denoting some function on $T^{2} / Z_{2}$. Note that the pull-back of this to D7 wrapping $K 3$ is identically zero, and that the effective FI constants on D3 near the D7 vanishes. Even if we chose another gauge such as

$$
B^{N S-N S} \sim f \wedge \mathrm{d} \alpha
$$

$B^{N S-N S}$ is odd under the orientifold projection and $f$ has to vanish at the four fixed points of $T^{2} / Z_{2}$, and in this orientifold limit the D7 branes are precisely located at these fixed points. Again the FI constants are not turned on D3 near D7, and the Coulomb branch moduli of D3's are intact.

Precise matching of moduli of branes wrapping 2-cycles in $K 3$ is a lot more involved problem, in part because on the IIB side D7 branes in question could have a worldvolume gauge bundle [49]. For this, we need a similar characterization of supersymmetric conditions on the heterotic side as well. General supersymmetry condition on D-branes in flux compactification of type IIB theory is by now understood fairly well [50, 51. It would be most interesting to translate this to the heterotic side and formulate the most general supersymmetric condition including the worldvolume field strengths in the presence of bulk flux.

\section{Summary}

We studied calibrated fivebranes in flux compactification of the heterotic string theory. Internal $H$-flux induces magnetic Coulomb potential for the internal part of the 
worldvolume, and a consistent supersymmetry condition in the absence of worldvolume field strength is found. The resulting calibration condition can have nontrivial solutions even without a topological winding number in a manner consistent with tadpole conditions. We apply this setup to a recent class of solution based on $T^{2}$ fibre-bundle over a conformally rescaled $K 3$, and found two distinct set of supersymmetric fivebranes. The fivebranes wrapping $T^{2}$ are supported by flux and also by a cyclic homotopy only, while the other is conventionally calibrated with respect to a Kähler form of $K 3$.

We also considered U-dual of this heterotic theory, realized as IIB orientifold $T^{2} / Z_{2} \times K 3$. Two types of calibrated fivebranes are found to be dual to D3 branes and certain D7 branes, respectively, and we matched some simple properties of the two sides. In particular, we noted how the non-extensive nature of $T^{2}$ winding number is mimicked by D3 branes in flux compactification of IIB theory, and performed a qualitative analysis.

Obviously this study still leaves much unaddressed. One interesting extension would involve precisely matching domain walls in IIB theory and those in the heterotic theory. In the latter, the domain wall interpolating two regions with different $T^{2}$ winding number of fivebranes, should be realized as a smooth configuration of fivebranes themselves. This is different from IIB side, where one find D3 branes ending on D5 or NS5.

Another interesting followup study would be how the low energy effective theory would look like in the presence of fivebranes wrapping the base $K 3$. For these, it is important to understand the worldvolume moduli fields better. However, this could turn out to be a difficult task since for consistency we should be careful to take into account the full effect of backreaction, as far as compact manifold goes.

\section{Acknowledgement}

We are indebted to Li-Sheng Tseng and Alessandro Tomasiello for useful comments, and also grateful to Tetsuji Kimura and Qing-Guo Huang for discussion. PY would 
like to thank USTC Shanghai Institute for Advanced Study, and the Center for Mathematical Physics for hospitality, and in particular to ICTP and the organizers of ICTP Workshop "String Vacua and Landscape" where much of the current work

was done. PY was supported in part by the Science Research Center Program of the Korea Science and Engineering Foundation through the Center for Quantum Spacetime(CQUeST) of Sogang University with grant number R11-2005-021. SK was supported in part by KOSEF Grant R010-2003-000-10391-0.

\section{References}

[1] L. Susskind, "The anthropic landscape of string theory," arXiv:hep-th/0302219.

[2] M. R. Douglas, "The statistics of string / M theory vacua," JHEP 0305, 046 (2003) arXiv:hep-th/0303194.

[3] S. Ashok and M. R. Douglas, "Counting flux vacua," JHEP 0401, 060 (2004) arXiv:hep-th/0307049.

[4] F. Denef and M. R. Douglas, "Distributions of flux vacua," JHEP 0405, 072 (2004) arXiv:hep-th/0404116.

[5] S. Kachru, R. Kallosh, A. Linde and S. P. Trivedi, "De Sitter vacua in string theory," Phys. Rev. D 68, 046005 (2003) arXiv:hep-th/0301240.

[6] S. B. Giddings, S. Kachru and J. Polchinski, "Hierarchies from fluxes in string compactifications," Phys. Rev. D 66, 106006 (2002) arXiv:hep-th/0105097.

[7] G. Lopes Cardoso, G. Curio, G. Dall'Agata, D. Lust, P. Manousselis and G. Zoupanos, "Non-Kaehler string backgrounds and their five torsion classes," Nucl. Phys. B 652, 5 (2003) arXiv:hep-th/0211118.

[8] J. P. Gauntlett, D. Martelli and D. Waldram, "Superstrings with intrinsic torsion," Phys. Rev. D 69, 086002 (2004) arXiv:hep-th/0302158.

[9] M. Gualtieri, "Generalized complex geometry," arXiv:math.dg/0401221. 
[10] M. Grana, R. Minasian, M. Petrini and A. Tomasiello, "Supersymmetric backgrounds from generalized Calabi-Yau manifolds," JHEP 0408, 046 (2004) arXiv:hep-th/0406137.

[11] M. Grana, R. Minasian, M. Petrini and A. Tomasiello, "Type II strings and generalized Calabi-Yau manifolds," Comptes Rendus Physique 5, 979 (2004) arXiv:hep-th/0409176.

[12] M. Grana, R. Minasian, M. Petrini and A. Tomasiello, "Generalized structures of $\mathrm{N}=1$ vacua," JHEP 0511, 020 (2005) arXiv:hep-th/0505212.

[13] M. Grana, "Flux compactifications in string theory: A comprehensive review," Phys. Rept. 423, 91 (2006) arXiv:hep-th/0509003.

[14] K. Becker and M. Becker, "M-Theory on Eight-Manifolds," Nucl. Phys. B 477, 155 (1996) arXiv:hep-th/9605053.

[15] K. Dasgupta, G. Rajesh and S. Sethi, "M theory, orientifolds and G-flux," JHEP 9908, 023 (1999) arXiv:hep-th/9908088.

[16] K. Becker and K. Dasgupta, "Heterotic strings with torsion," JHEP 0211, 006 (2002) arXiv:hep-th/0209077.

[17] K. Becker, M. Becker, K. Dasgupta and P. S. Green, "Compactifications of heterotic theory on non-Kaehler complex manifolds. I," JHEP 0304, 007 (2003) arXiv:hep-th/0301161.

[18] K. Becker, M. Becker, K. Dasgupta, P. S. Green and E. Sharpe, "Compactifications of heterotic strings on non-Kaehler complex manifolds. II," Nucl. Phys. B 678, 19 (2004) arXiv:hep-th/0310058.

[19] P. S. Aspinwall and R. Kallosh, "Fixing all moduli for M-theory on K3 x K3," JHEP 0510, 001 (2005) arXiv:hep-th/0506014.

[20] K. Becker and L. S. Tseng, "Heterotic flux compactifications and their moduli," Nucl. Phys. B 741, 162 (2006) arXiv:hep-th/0509131. 
[21] D. J. Gross, J. A. Harvey, E. J. Martinec and R. Rohm, "The Heterotic String," Phys. Rev. Lett. 54, 502 (1985).

[22] A. Strominger, "Superstrings With Torsion," Nucl. Phys. B 274, 253 (1986).

[23] M. B. Green and J. H. Schwarz, "Anomaly Cancellation In Supersymmetric D=10 Gauge Theory And Superstring Theory," Phys. Lett. B 149, 117 (1984).

[24] E. A. Bergshoeff and M. de Roo, "The Quartic Effective Action Of The Heterotic String And Supersymmetry," Nucl. Phys. B 328, 439 (1989).

[25] T. Kimura and P. Yi, "Comments on heterotic flux compactifications," arXiv:hep-th/0605247.

[26] S. Ivanov and G. Papadopoulos, "A no-go theorem for string warped compactifications," Phys. Lett. B 497, 309 (2001) arXiv:hep-th/0008232.

[27] J. X. Fu and S. T. Yau, "The theory of superstring with flux on non-Kaehler manifolds and the complex Monge-Ampere equation," arXiv:hep-th/0604063

[28] K. Becker, M. Becker, J. X. Fu, L. S. Tseng and S. T. Yau, "Anomaly cancellation and smooth non-Kaehler solutions in heterotic string theory," arXiv:hep-th/0604137.

[29] J. Polchinski, String Theory, 2 vols., Cambridge University Press, 1998.

[30] J. M. Bismut, " A Local Index Theorem for Non-Kähler Manifolds," Math. Ann. 284 (1989) 681.

[31] G. Lopes Cardoso, G. Curio, G. Dall'Agata and D. Lust, "BPS action and superpotential for heterotic string compactifications with fluxes," JHEP 0310, 004 (2003) arXiv:hep-th/0306088.

[32] C. M. Hull, "Anomalies, Ambiguities And Superstrings," Phys. Lett. B 167, 51 (1986).

[33] C. M. Hull, "Compactifications Of The Heterotic Superstring," Phys. Lett. B 178, 357 (1986). 
[34] A. Sen, "(2,0) Supersymmetry And Space-Time Supersymmetry In The Heterotic String Theory," Nucl. Phys. B 278, 289 (1986).

[35] M. J. Duff, J. T. Liu and R. Minasian, "Eleven-dimensional origin of string / string duality: A one-loop test," Nucl. Phys. B 452, 261 (1995) arXiv:hep-th/9506126.

[36] P. Horava and E. Witten, "Heterotic and type I string dynamics from eleven dimensions," Nucl. Phys. B 460, 506 (1996) arXiv:hep-th/9510209.

[37] P. Horava and E. Witten, "Eleven-Dimensional Supergravity on a Manifold with Boundary," Nucl. Phys. B 475, 94 (1996) arXiv:hep-th/9603142.

[38] J. Gutowski, G. Papadopoulos and P. K. Townsend, "Supersymmetry and generalized calibrations," Phys. Rev. D 60, 106006 (1999) arXiv:hep-th/9905156.

[39] A. Sen, "F-theory and Orientifolds," Nucl. Phys. B 475, 562 (1996) arXiv:hep-th/9605150.

[40] C. Vafa, "Evidence for F-Theory," Nucl. Phys. B 469, 403 (1996) arXiv:hep-th/9602022.

[41] T. H. Buscher, "Path Integral Derivation of Quantum Duality in Nonlinear Sigma Models," Phys. Lett. B 201, 466 (1988).

[42] E. Witten, "String Theory Dynamics In Various Dimensions," Nucl. Phys. B 443, 85 (1995) arXiv:hep-th/9503124.

[43] J. Polchinski and E. Witten, "Evidence for Heterotic - Type I String Duality," Nucl. Phys. B 460, 525 (1996) arXiv:hep-th/9510169.

[44] E. Goldstein and S. Prokushkin, "Geometric model for complex non-Kaehler manifolds with SU(3) structure," Commun. Math. Phys. 251, 65 (2004) arXiv:hep-th/0212307.

[45] S. Gukov, C. Vafa and E. Witten, "CFT's from Calabi-Yau fourfolds," Nucl. Phys. B 584, 69 (2000) [Erratum-ibid. B 608, 477 (2001)] arXiv:hep-th/9906070. 
[46] M. Bershadsky, C. Vafa and V. Sadov, "D-Branes and Topological Field Theories," Nucl. Phys. B 463, 420 (1996) arXiv:hep-th/9511222.

[47] P. S. Aspinwall and R. Kallosh, JHEP 0510, 001 (2005) arXiv:hep-th/0506014.

[48] E. Witten, "Small Instantons in String Theory," Nucl. Phys. B 460, 541 (1996) arXiv:hep-th/9511030.

[49] J. Gomis, F. Marchesano and D. Mateos, "An Open String Landscape," JHEP 0511, 021 (2005) arXiv:hep-th/0506179.

[50] L. Martucci and P. Smyth, "Supersymmetric D-Branes And Calibrations On General N = 1 Backgrounds," JHEP 0511, 048 (2005) arXiv:hep-th/0507099.

[51] L. Martucci, "D-branes on general $N=1$ backgrounds: Superpotentials and D-terms," arXiv:hep-th/0602129. 